\title{
Resource Allocation in Fading Wireless Ad-Hoc Networks with Temporally Correlated Loss
}

\author{
Lynn Zheng Homayoun Yousefi'zadeh Hamid Jafarkhani \\ Department of EECS \\ University of California, Irvine
}

[llzheng, hyousefi,hamidj] @uci.edu

\begin{abstract}
Addressing the tradeoff between the QoS and consumed power is a critical issue for wireless ad-hoc networks. The loss observed in such networks is often temporally correlated. This paper examines an optimal scheme to maximize the aggregate data rate of wireless ad-hoc networks under the power and loss constraints. In order to properly model temporally correlated loss observed in a fading wireless channel, we propose the use of finite-state Markov chains. Details of fading statistics of signal-tointerference ratio (SIR), an important indicator of transmission quality, are presented. We also analyze the impacts of enforcing power, block-loss probabilities, and data rates constraints.

Index Terms - Wireless Ad-Hoc Networks, Rayleigh Fading Channel, Throughput Maximization, QoS, Markov Chain Model, Reed-Solomon Channel Coder, Adaptive Modulation.
\end{abstract}

\section{INTRODUCTION}

Recent proliferation of wireless devices has greatly facilitated access and exchange of information. Ad-hoc networks are a special class of wireless networks where there is no such fixed infrastructure as base stations for allocating channels, controlling usage, or provisioning of services. Rather, they need to be adaptively self-organizing. Any node in an ad-hoc network can transmit, receive, or relay signals. Optimal allocation of resources under the power constraint is critical both for increased utilization of the limited wireless spectrum and for longer battery life of the mobile devices. Such an optimal allocation introduces an intelligent way of providing the desired level of quality of service (QoS) under the power constraints in wireless environments.

Due to the openness of transmission media, communication over a wireless link is prone to interferences from other links in addition to noise. Often times, the former factor has a much greater effect than the latter. While increasing the transmission power of a user in an ad-hoc network will make the outgoing link more reliable, it also shortens battery life and causes interference to other users. Hence, the observed signalto-interference ratio (SIR) at the receiver is introduced to capture the tradeoff. Further, considering the mobility of the nodes in an ad-hoc network, links are subject to the Doppler spread. Although temporal correlation between signals cannot be neglected, to date only few articles have utilized accurate models of capturing temporally correlated loss of the wireless channel.

Among the common models proposed to characterize a flat Rayleigh fading channel, one can find the Gilbert-Elliot channel model of [4] followed by the model of [9] extending the original model to a finite state Markov chain model. In [8], Tan et al. examined the validity of an amplitude-based finite-state Markov chain model under the assumption that the use of the first-order and second-order fading statistics can potentially improve the system performance. Yousefi'zadeh et al. considered the twostate Gilbert-Elliott loss model to capture temporally correlated loss when optimizing total power consumption in end-to-end transmission of multimedia content over wireless fading channels [10]. Hayajneh et al. [5] proposed a game-theoretic power control algorithm for wireless channels. Relying on Geometric Programming, a special case of convex optimization, Chiang et al. [2] solved a set of resource allocation problems for QoS provisioning in wireless ad-hoc networks. However, neither paper took into account temporally correlated loss and time-varying characteristics of the wireless channel.

The main contribution of our work is integrating a finitestate Markov chain model into resource allocation problems in wireless ad-hoc networks without incurring prohibitive overhead. Relying on more accurate characterization of wireless channels specifically a Rayleigh fading channel model, we propose the use of temporally correlated block-loss probabilities in measuring QoS. Our approach is straightforward to extend to other channel models such as Ricean and Nakagami fading channels. The rest of this paper is organized as follows. In Section II, we assess the underlying system model. More specifically, first we analyze first-order and second-order statistics of SIR. Second, we express the symbol error rate as a function of the average received SIR. We also examine the blockloss probability based on symbol error rate and a finite-state Markov chain loss model. In Section III, we formulate a resource allocation problem aimed at maximizing the overall system throughput subject to power and loss constraints. Detailed problem formulation and solution are presented. Section IV provides simulation results. Finally, Section V concludes this work and discusses future work.

\section{System Model Assessment}

In this section, we assess the system model utilized for our problem. From the perspective of a system, we seek to maximize the overall throughput under the constraints related to powers, minimum link data rates, and per link block-loss probabilities. 


\section{A. Analysis of Received Signal-to-Interference Ratio}

Consider $n$ wireless links, labeled $L_{1}, \ldots, L_{n}$, on which transmission powers are $P_{1}, \ldots, P_{n}$, respectively. Link $i$ is associated with the $i$-th transmitter/receiver pair. At the end of link $i$, the power at receiver $i$ is given by

$$
G_{i i}(t) P_{i}(t) F_{i i}(t)
$$

Similarly, interfering signals from all of the other links on which $P_{j}$ 's $(i \neq j)$ are transmitted are given by

$$
G_{i j}(t) P_{j}(t) F_{i j}(t)
$$

The nonnegative number $G_{i j}(t)$ represents the path gain in the absence of fading from the transmitter of link $j$ to the receiver of link $i$ at time $t . G_{i j}(t)$ captures such factors as path loss, shadowing, antenna gain, and so on. $F_{i j}(t)$ is the fading factor between the transmitter of link $j$ and the receiver of link $i$. The instantaneous signal-to-interference ratio at time $t$ for link $i$ determines the quality of the received signal and is defined as

$$
S I R_{i}(t)=\frac{G_{i i}(t) P_{i}(t) F_{i i}(t)}{\sum_{j \neq i} G_{i j}(t) P_{j}(t) F_{i j}(t)+N_{i}(t)}
$$

where $N_{i}(t)$ represents the white Gaussian noise on link $i$. We note that while the work of [2] assumes identical and independent distribution of all of the fading factors, this assumption is not necessarily true in wireless channels. Instead, the channel is temporally-correlated, i.e. there is a correlation between $F_{i j}(t)$ and $F_{i j}(t+\Delta t)$ where $\Delta t$ is a given time shift. In order to capture the correlation of the channel, we make few realistic assumptions as follows. First, compared with interferences from other users, noise is negligible and thus may be accurately ignored. Second, it is reasonable to assume that the fading factors in interfering signals $F_{i j}$ 's where $i \neq j$, have identical and independent distributions. We note that the latter assumption may be relaxed relying on a similar discussion to the one furnished next for the fading components $F_{i i}$. Third, $F_{i j}$ 's have unit means so long as $G_{i j}$ 's are appropriately scaled to reflect variations from this assumption. Fourth, when the wireless channel varies slowly with respect to symbol interval, $P_{i}(t)$ and $G_{i j}(t)$ can be viewed as constants and $F_{i j}(t)$ as a random variable within the symbol duration. Based on the above assumptions, we define the average signal-to-interference ratio $S I R_{i}$ as

$$
S I R_{i}=\frac{E\left[G_{i i} P_{i} F_{i i}(t)\right]}{E\left[G_{i j} P_{j} F_{i j}(t)\right]}=\frac{G_{i i} P_{i} F_{i i}}{\sum_{j \neq i} G_{i j} P_{j}}
$$

where $E[$.$] denotes the expectation operator and E\left[F_{i i}(t)\right]=$ $F_{i i}$. Hence, it suffices to examine the distribution of $F_{i i}(t)$ in order to obtain fading statistics of $S I R_{i}$. We rely on the socalled Rayleigh model with the fading factor $\alpha$ to relate the output of a wireless noisy channel to its input. The output signal of such a channel $S_{o}$ and its input $S_{i}$ can be related by

$$
S_{o}=\alpha S_{i}+N
$$

where $N$ represents the white Gaussian noise. It is well established that $r=|\alpha|$ has a marginal Rayleigh distribution function in the form of

$$
p(r)=\frac{r e^{-r^{2} / 2 \mu^{2}}}{\mu^{2}}, r \geq 0
$$

where $\mu^{2}$ equals to half of the average power of all of the multipath components. In order to properly characterize the temporally correlated loss behavior of the channel, we also need the associated bivariate joint probability distribution function given below.

$$
\begin{aligned}
p\left(r_{i}, r_{j}\right)= & \frac{r_{i} r_{j}}{\mu^{2}\left(1-\lambda^{2}\right)} \exp \left(-\frac{r_{i}^{2}+r_{j}^{2}}{2 \mu\left(1-\lambda^{2}\right)}\right) I_{0}\left(\frac{r_{i} r_{j} \lambda}{\mu\left(1-\lambda^{2}\right)}\right) \\
& r_{i} \geq 0, r_{j} \geq 0
\end{aligned}
$$

where $I_{0}$ is the zero-order modified Bessel function of the first kind given by

$$
I_{0}(z)=\sum_{l=0}^{\infty} \frac{z^{2 l}}{2^{2 l}(l !)^{2}}, \quad z \geq 0
$$

Further,

$$
\mu^{2}=\frac{E\left(r_{i}^{2}\right)}{2}=\frac{E\left(r_{j}^{2}\right)}{2}
$$

and

$$
\lambda=J_{0}\left(2 \pi f_{m} \tau\right)
$$

with $J_{0}$ representing the zero-order Bessel function of the first kind defined as

$$
J_{0}(z)=\sum_{l=0}^{\infty} \frac{(-1)^{l} z^{2 l}}{2^{2 l}(l !)^{2}}, \quad z \geq 0
$$

Finally, $f_{m}$ and $\tau$ represent maximum frequency shift resulting from the Doppler effect and symbol duration, respectively. Reference [6] includes a detailed derivation of both distribution functions. Considering the fact that $F_{i i}$ is defined as $F_{i i}=r_{i}^{2}=\left|\alpha_{i}\right|^{2}$, we are interested in the distribution of $|\alpha|^{2}$. In order to calculate the distribution of $|\alpha|^{2}$, we first note that Equation (9) implies $\mu=\frac{1}{\sqrt{2}}$ under the assumption that $F_{i i}$ 's have unit means. Consequently, utilizing Equations (6) and (7), we conclude that $F_{i i}$ has a marginal probability density function and a bivariate joint probability density function as shown in Equation (12) and Equation (13), respectively.

$$
\begin{gathered}
p\left(F_{i i}\right)=\frac{e^{-F_{i i} / 2 \mu^{2}}}{2 \mu^{2}}, F_{i i} \geq 0 \\
p\left(F_{i i}, F_{j j}\right)=\frac{1}{4 \mu^{2}\left(1-\lambda^{2}\right)} \exp \left(-\frac{F_{i i}+F_{j j}}{2 \mu\left(1-\lambda^{2}\right)}\right) I_{0}\left(\frac{\sqrt{F_{i i} F_{j j} \lambda}}{\mu\left(1-\lambda^{2}\right)}\right) \\
i \neq j, \quad F_{i i} \geq 0, \quad F_{j j} \geq 0
\end{gathered}
$$

Having specified the probability density functions, we can now focus on capturing temporally correlated loss of the fading Rayleigh channel. We propose partitioning and modeling such an analog channel with a digital Markov chain model. A review of the literature reveals that there are numerous articles on partitioning the received SNR or SIR. References [9] and [8] are of special interest to us among those articles. In our work, we apply the fundamental idea of partitioning to the probability 
density function (pdf) of $F_{i i}$ in order to model the underlying Rayleigh fading channel with a finite-state Markov chain. As illustrated by Fig. 1, suppose that pdf of $F_{i i}$ is partitioned into $S$ finite intervals starting at zero and ending at $I N F$. INF is a large real number satisfying

$$
\int_{0}^{I N F} p\left(F_{i i}\right) d F_{i i}=1-\delta
$$

where $\delta$, e.g. $10^{-5}$, indicates the probability of not representing a value of the pdf of $F_{i i}$ by any state. Setting $I N F=9$ guarantees that $99.99 \%$ of the pdf values are covered. In our work,

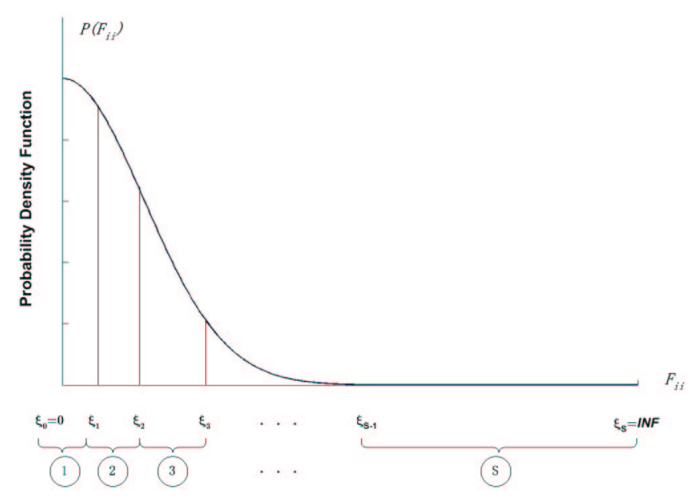

Fig. 1. An illustration of finite interval partitioning of an arbitrary pdf.

we propose the use of an equally probable partitioning, i.e. we want to find a set of thresholds $\left\{\xi_{1}, \cdots, \xi_{S}\right\}$, such that

$$
\begin{aligned}
& \xi_{0}=0 \\
& \xi_{S}=I N F \\
& \int_{\xi_{s-1}}^{\xi_{s}} p\left(F_{i i}\right) d F_{i i}=\frac{1-\delta}{S}, s=1, \ldots, S
\end{aligned}
$$

As an alternative to an equally probable partitioning approach, the partitioning thresholds can be measured from the natural burst lengths of a wireless channel. The thresholds allow us to find $F_{i i, s}$, the representative value of the pdf of $F_{i i}$ in state $s$, by

$$
F_{i i, s}=\int_{\xi_{s-1}}^{\xi_{s}} F_{i i} p\left(F_{i i}\right) d F_{i i}, \quad s=1, \ldots, S
$$

Therefore, the corresponding representative values of $S I R_{i}$ 's are given by

$$
S I R_{i, s}=\frac{G_{i i} P_{i} F_{i i, s}}{\sum_{j \neq i} G_{i j} P_{j}}, \quad s=1, \ldots, S
$$

The temporal dynamics of the Markov chain are determined by a matrix of one-step-transition probabilities $\Pi=\left[\pi_{s r}\right]_{S \times S}$. Since we are working with slow-fading channels, it is reasonable to consider only transitions between neighboring states or staying in the same state [9]. According to Bayes theorem, the probability of currently being in state $r$ given having previously been in state $s$ can be computed as

$$
\tilde{\pi}_{s r}=\frac{\int_{\xi_{r-1}}^{\xi_{r}} \int_{\xi_{s-1}}^{\xi_{s}} p\left(F_{i i}, F_{j j}\right) d F_{i i} d F_{j j}}{\int_{\xi_{s-1}}^{\xi_{s}} p\left(F_{i i}\right) d F_{i i}}
$$

where $r \in\{s, s \pm 1\}$ and for $1 \leq r, s \leq S$. Due to the finite value of the error introduced by the model and the transitioning assumption, we must modify the transition matrix such that each row adds up to one. This can be done by uniformly scaling each row by its sum as

$$
\pi_{s r}=\frac{\tilde{\pi}_{s r}}{\sum_{s=1}^{S} \tilde{\pi}_{s r}}
$$

\section{B. Compensation of Temporally Correlated Loss}

We start the discussion of this section by introducing a per link per state approximation of $M_{i, s}$ the number of signal point constellations in terms of per link per state bit error rate $B E R_{i, s}$ and per link per state symbol to interference ratio $S I R_{i, s}$. Expanding adaptive modulation results of Chung et al. [3] for MQAM modulation to our finite state Markov chain model, we note that $B E R_{i, s}$ can be closely approximated as

$$
B E R_{i, s}=0.2 \exp \left(\frac{-1.6 S I R_{i, s}}{M_{i, s}-1}\right)
$$

assuming $\log _{2} M_{i, s} \geq 2$. For a slow fading channel, per state symbol error rates can be accurately approximated as

$$
S E R_{i, s}=1-\left(1-B E R_{i, s}\right)^{\log _{2} M_{i, s}} \simeq B E R_{i, s} \log _{2} M_{i, s}
$$

Per link data rate $R_{i}$ can further be expressed as

$$
R_{i}=\sum_{s=1}^{S} \zeta_{s} R_{i, s}=\frac{1}{T} \sum_{s=1}^{S} \zeta_{s} \log _{2} M_{i, s}
$$

where $\frac{1}{T}$ is the baseband bandwidth and $\zeta_{s}$ with $s \in\{1, \cdots, S\}$ the steady state probability of being in state $s$ can be calculated from the transition probabilities of a given Markov chain. We note that in the case of equally probable partitioning, $\zeta_{s}=\frac{1}{S}$ for all $s$.

In order to compensate for the loss effect of the wireless channel, we propose the use of Reed-Solomon (RS) channel coders. An RS channel coder $R S(b, k)$ converts $k$ symbols into a $b$-symbol block by appending $(b-k)$ parity symbols. Such a channel coder is able to correct as many as $t C=\left\lfloor\frac{b-k}{2}\right\rfloor$ symbol errors in a block. In the calculation of the block-loss probability, we consider using a 2-state Markov chain model described in Fig. 2, and a 3-state Markov chain model shown in Fig. 3. Intuitively, the 3-state model is more accurate than its 2-state counterpart, at the cost of higher computational complexity. Equations (12) through (15), (18), and (19) in Section II-A have given details of deriving transition probabilities $\tilde{\pi}_{s r}$ 's from marginal and joint probability density functions.

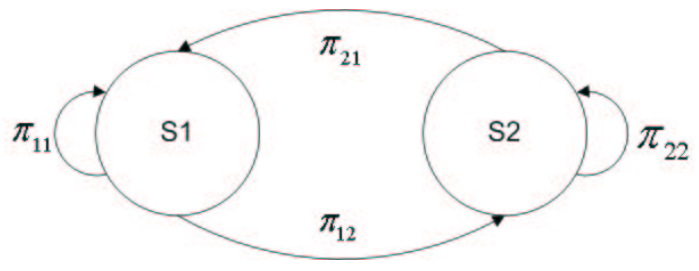

Fig. 2. An illustration of the 2-state Markov chain model. 


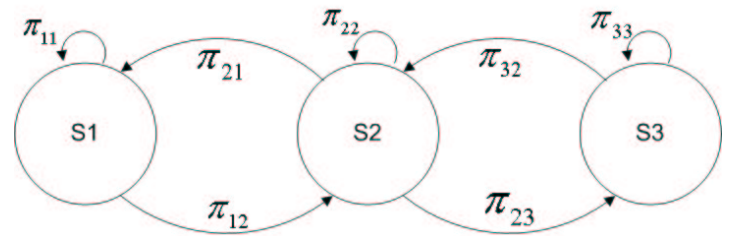

Fig. 3. An illustration of the 3-state Markov chain model.

Let $\varphi(b, k, s)$ denote the probability of receiving exactly $k$ symbols from $b$ symbols and winding up in state $s$. The probability of receiving exactly $k$ symbols from a $b$-symbol block is given by

$$
\varphi(b, k)=\sum_{s=1}^{S} \varphi(b, k, s)
$$

If a user receives at least $b-t C$ symbols correctly from $b$ transmitted symbols, the whole block is recoverable. Hence, the block-loss probability is expressed as

$$
\Psi=1-\sum_{k=b-t C}^{b} \varphi(b, k)
$$

Next, we apply the block loss probability results to the 2-state and 3-state Markov chains. First, we consider using a 2-state Markov chain model. $\varphi(b, k, 1)$ and $\varphi(b, k, 2)$ indicate the probabilities of receiving exactly $k$ symbols from $b$ symbols and winding up in state 1 and state 2 , respectively. Thus,

$$
\varphi(b, k)=\varphi(b, k, 1)+\varphi(b, k, 2)
$$

In accordance with the underlying Markov chain model, $\varphi(b, k, 1)$ and $\varphi(b, k, 2)$ can be computed recursively. The expressions are given below.

$$
\begin{aligned}
\varphi(b, k, 1) & =S E R_{1}\left[\pi_{11} \varphi(b-1, k, 1)+\pi_{21} \varphi(b-1, k, 2)\right] \\
& +\left(1-S E R_{1}\right)\left[\pi_{11} \varphi(b-1, k-1,1)\right. \\
& \left.+\pi_{21} \varphi(b-1, k-1,2)\right]
\end{aligned}
$$

and

$$
\begin{aligned}
\varphi(b, k, 2) & =S E R_{2}\left[\pi_{12} \varphi(b-1, k, 1)+\pi_{22} \varphi(b-1, k, 2)\right] \\
& +\left(1-S E R_{2}\right)\left[\pi_{12} \varphi(b-1, k-1,1)\right. \\
& \left.+\pi_{22} \varphi(b-1, k-1,2)\right]
\end{aligned}
$$

for $b \geq k>0, S E R_{s}$ denoting the symbol error rate in state $s$, and the initial conditions

$$
\begin{aligned}
& \varphi(0,0,1)=\varphi(0,0,2)=\frac{1}{2} \\
& \varphi(1,0,1)=S E R_{1}\left[\pi_{11} \varphi(0,0,1)+\pi_{21} \varphi(0,0,2)\right] \\
& \varphi(1,0,2)=S E R_{2}\left[\pi_{12} \varphi(0,0,1)+\pi_{22} \varphi(0,0,2)\right] \\
& \varphi(1,1,1)=\left(1-S E R_{1}\right)\left[\pi_{11} \varphi(0,0,1)+\pi_{21} \varphi(0,0,2)\right] \\
& \varphi(1,1,2)=\left(1-S E R_{2}\right)\left[\pi_{12} \varphi(0,0,1)+\pi_{22} \varphi(0,0,2)\right]
\end{aligned}
$$

Similarly, if a 3-state Markov chain model is used, we have

$$
\varphi(b, k)=\varphi(b, k, 1)+\varphi(b, k, 2)+\varphi(b, k, 3)
$$

where

$$
\begin{aligned}
\varphi(b, k, 1) & =S E R_{1}\left[\pi_{11} \varphi(b-1, k, 1)+\pi_{21} \varphi(b-1, k, 2)\right] \\
& +\left(1-S E R_{1}\right)\left[\pi_{11} \varphi(b-1, k-1,1)\right. \\
& \left.+\pi_{21} \varphi(b-1, k-1,2)\right]
\end{aligned}
$$

$$
\begin{aligned}
\varphi(b, k, 2) & =S E R_{2}\left[\pi_{12} \varphi(b-1, k, 1)+\pi_{22} \varphi(b-1, k, 2)\right. \\
& \left.+\pi_{32} \varphi(b-1, k, 3)\right] \\
& +\left(1-S E R_{2}\right)\left[\pi_{12} \varphi(b-1, k-1,1)\right. \\
& \left.+\pi_{22} \varphi(b-1, k-1,2)+\pi_{32} \varphi(b-1, k, 3)\right]
\end{aligned}
$$

and

$$
\begin{aligned}
\varphi(b, k, 3) & =S E R_{3}\left[\pi_{23} \varphi(b-1, k, 2)+\pi_{33} \varphi(b-1, k, 3)\right] \\
& +\left(1-S E R_{3}\right)\left[\pi_{23} \varphi(b-1, k-1,2)\right. \\
& \left.+\pi_{33} \varphi(b-1, k-1,3)\right]
\end{aligned}
$$

for $b \geq k>0$ and the initial conditions

$$
\begin{aligned}
\varphi(0,0,1) & =\varphi(0,0,2)=\varphi(0,0,3)=\frac{1}{3} \\
\varphi(1,0,1) & =S E R_{1}\left[\pi_{11} \varphi(0,0,1)+\pi_{21} \varphi(0,0,2)\right] \\
\varphi(1,0,2) & =S E R_{2}\left[\pi_{12} \varphi(0,0,1)+\pi_{22} \varphi(0,0,2)\right. \\
& \left.+p_{32} P(0,0,3)\right] \\
\varphi(1,0,3) & =S E R_{3}\left[\pi_{23} \varphi(0,0,2)+\pi_{33} \varphi(0,0,3)\right] \\
\varphi(1,1,1) & =\left(1-S E R_{1}\right)\left[\pi_{11} \varphi(0,0,1)+\pi_{21} \varphi(0,0,2)\right] \\
\varphi(1,1,2) & =\left(1-S E R_{2}\right)\left[\pi_{12} \varphi(0,0,1)+\pi_{22} \varphi(0,0,2)\right. \\
& \left.+\pi_{32} \varphi(0,0,3)\right] \\
\varphi(1,1,3) & =\left(1-S E R_{3}\right)\left[\pi_{23} \varphi(0,0,2)+\pi_{33} \varphi(0,0,3)\right]
\end{aligned}
$$

At the end of this section, a discussion of channel state information (CSI) is in order. We consider two scenarios. In the first scenario, we assume that CSI is known at the encoder and hence the transmitter. Therefore, the calculations of optimal per state per channel $B E R_{i, s}$ in Equation (20) can be carried out. In the second scenario, we assume that CSI is unknown at the encoder/transmitter. We note that in the latter scenario, the set of per link per state parameters $M_{i, s}$ are reduced to a set of per link parameters $M_{i}$. Although we consider the effects of per state per channel $B E R_{i, s}$ in the latter case, we use the expectations of the per state quantities in the calculation of $B E R_{i, s}$, namely $\overline{B E R}_{i}=\sum_{s=1}^{S} \zeta_{s} B E R_{i, s}$ and $\overline{S I R}_{i}=\sum_{s=1}^{S} \zeta_{s} S I R_{i, s}$.

\section{Resource Allocation Problem}

In this section, we formulate our problem and provide a solution to it. Our goal is to examine optimal ways of allocating powers in order to achieve maximum aggregate data rate subject to block-loss probability requirements among other constraints. Specifically in a network with $n$ links, we aim at maximizing the system throughput.

$$
\begin{array}{ll}
\max _{P_{i}, M_{i, s}} & R_{\text {total }}=\sum_{i=1}^{n} R_{i} \\
\text { Subject To: } & \Psi_{i} \leq \Psi_{i, u b} \quad \forall i \\
& R_{i, s} \geq R_{i, s, l b} \quad \forall i, s \\
& P_{i} \leq P_{i, u b} \quad \forall i
\end{array}
$$

We note that in the formulation above, $P_{i}$ 's and $M_{i, s}$ 's are the decision variables. Further, we note that the SER parameters of the Markov chain discussion of Section II-B appear in the form of a set of per link per state parameters. We also note that while our formulation is a throughput maximization subject to power constraints, it can be converted to a power minimization subject to throughput constraints by interchanging the role of the objective function (34) and the constraint function (37). Expressions 
(4), (20), and (22) show that the data rates $R_{i}$ 's are related with per link per state constellation sizes $M_{i, s}$. Therefore, the objective function (34) is to be optimized over all feasible powers and constellation sizes. The first set of constraints (35) is the maximum allowable block-loss probability on each link. Constraint set (36) is enforced so that the approximation of Equation (20) under Equation (22) holds. Same as the objective function, this set is related with $M_{i, s}$ 's. The last set of constraints (37) indicates regulatory or system limitations on transmission powers. As shown by Equations (20) through (33) in Section II-B, $\Psi_{i}$ 's are essentially functions of per link per state $S E R_{i, s}$ 's, hence of $P_{i}$ 's as well as $M_{i, s}$ 's.

Next, we provide a discussion of solving the optimization problem formulated by (34) along with the constraint sets (35), (36), and (37). Relying on the Lagrangian theory, we convert the problem in its standard form to an optimization problem without constraints. We define the Lagrangian function of the original problem as

$$
\begin{aligned}
L= & -\sum_{i=1}^{n} R_{i}+\sum_{i=1}^{n} \lambda_{i}\left(\Psi_{i}-\Psi_{i, u b}\right) \\
& +\sum_{s=1}^{n} \sum_{i=1}^{n} \mu_{i, s}\left(-R_{i, s}+R_{i, s, l b}\right) \\
& +\sum_{i=1}^{n} \nu_{i}\left(P_{i}-P_{i, u b}\right)
\end{aligned}
$$

where the parameters $\lambda_{i}, \mu_{i, s}$, and $\nu_{i}$ are the Lagrange multipliers in the Lagrangian Equation (38). The unconstrained minimization problem is defined as

$$
\begin{aligned}
\min _{P_{i}, M_{i, s}} L & =\min _{M_{i, s}, P_{i}}\left\{-\sum_{i=1}^{n} R_{i}\right. \\
& +\sum_{i=1}^{n} \lambda_{i}\left(\Psi_{i}-\Psi_{i, u b}\right) \\
& +\sum_{s=1}^{S} \sum_{i=1}^{n} \mu_{i, s}\left(-R_{i, s}+R_{i, s, l b}\right) \\
& \left.+\sum_{i=1}^{n} \nu_{i}\left(P_{i}-P_{i, u b}\right)\right\}
\end{aligned}
$$

\section{Conditions of Optimality: Constraint Qualifications}

We now investigate the existence of necessary and sufficient optimality conditions also known as constraint qualifications. For our unconstrained minimization problem of (39), the constraint qualifications are expressed in terms of Lagrange multiplier theory [1]. They revolve around conditions under which Lagrange multiplier vectors satisfying the following conditions are guaranteed to exist for a local optimum $\Omega^{*}=\left\{P_{i}{ }^{*}, M_{i, s}^{*}\right\}$ that satisfies

$$
\nabla L\left(\Omega^{*}\right)=0
$$

where $\nabla L=\left[\frac{\partial L}{\partial P_{i}}, \frac{\partial L}{\partial M_{i, s}}\right]$.

Although the constraints (35) cannot be expressed in a closed form, we are still able to solve the problem by deploying Sequential Quadratic Programming (SQP) and line search techniques. In SQP, the necessary conditions for optimality are represented by the Karush-Kuhn-Tucker (KKT) conditions described as the collection of Equation (40) and the following relationships.

$$
\begin{array}{ll}
\lambda_{i}{ }^{*}\left(\Psi_{i}{ }^{*}-\Psi_{i, u b}\right)=0 & \forall i \\
\mu_{i, s}{ }^{*}\left(-R_{i, s}{ }^{*}+R_{i, s, l b}\right)=0 & \forall i, s \\
\nu_{i}{ }^{*}\left(P_{i}{ }^{*}-P_{i, u b}\right)=0 & \forall i \\
\lambda_{i}{ }^{*}, \mu_{i, s}{ }^{*}, \nu_{i}{ }^{*} \geq 0 & \forall i, s
\end{array}
$$

where $\lambda_{i}{ }^{*}, \mu_{i, s}{ }^{*}$, and $\nu_{i}{ }^{*}$ are Lagrange multipliers at the local optimum. Positive multipliers indicate active constraints. A variant of the quasi-Newton method can then be used to iteratively find the solution to the optimization problem [7]. This is equivalent to solving a quadratic estimation of the problem in every iteration.

We end this section by presenting an analysis of the computational complexity for the approach described above. The time complexity of solving the problem of (40) is $\mathcal{O}(I d \log d)$ where $I$ indicates the number of iterations and $d$ indicates the degree of the quadratic estimation. For moderate values of $I$, the complexity results are hence quite good compared to other recursive optimization approaches such as dynamic programming introducing a time complexity in the order of $\mathcal{O}\left(d^{2}\right)$.

\section{Simulation Results}

In this section, we present an example to show how to maximize system throughput under power constraints. Let us consider a simple four node network. As shown in Fig. 4, the network consists of 4 nodes $A, B, C$ and $D$, and 4 links $L_{1}$, $L_{2}, L_{3}$, and $L_{4}$. Each link indicates a transmitter/receiver pair. Note that a node can be a transmitter and/or receiver on multiple links. We note that in the case of simultaneously transmitting on multiple links, the power of a node is split on the outgoing links proportionally. Originally, nodes $A$ and $D$ are separated by a distance of $20 \mathrm{~m}$, and so are $B$ and $C$. By geometry the distance of each single hop is $10 \sqrt{2} \mathrm{~m}$. In our experiments, we allow node $A$ to move across the horizontal axis $x$ both toward and away from node $D$. We indicate the position of node $A$ from a reference point by $x$. We select the reference point to be the middle of diagonal line connecting nodes $B$ and $C$. Hence, the original position of node $A$ is indicated by $x=10$. As explained earlier, we consider two scenarios in which CSI is known and not known, respectively. In our simulations, each

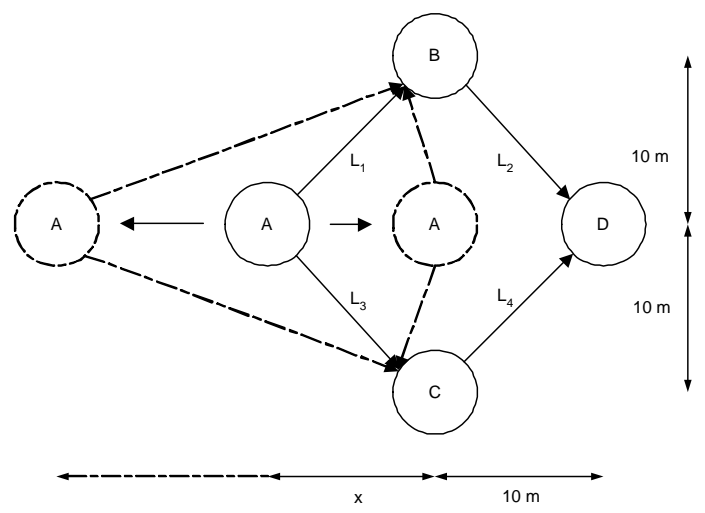

Fig. 4. An illustration of the network topology used in the simulation task.

link has a maximum transmission power of $1 \mathrm{~W}$. All nodes are using adaptive M-QAM modulation representing a symbol with $\log _{2} M_{i, s}$ bits per link per state. The baseband bandwidth for each link is $10 \mathrm{kHz}$ and the minimum data rate for each link is $20 \mathrm{kbps}$ under the conditions of Equation (20) and Equation (22). We set a maximum allowable block-loss probability of 0.1 on each link. With the exception of $G_{12}, G_{34}$, the gains for each link are computed as $G_{i i}=\frac{1}{d_{i i}^{4}}$ and $G_{i j}=\frac{\eta}{d_{i j}^{4}}$ for $i \neq j$, where $d_{i j}$ represents propagation path length from the transmitter of link $j$ to the receiver of link $i$. The factor $\eta$ can be viewed 
as the power falloff with frequency in an FDMA system, or the spreading gain in a CDMA system. It is set as $\eta=0.005$ in our simulations. The gains for $G_{12}, G_{34}$ are set to 0 since it is assumed that a node does not transmit to itself. This gives the following gain matrix in terms of $x$.

$G=\left[\begin{array}{llll}1 /\left(x^{2}+100\right)^{2} & 0 & \eta /\left(x^{2}+100\right)^{2} & \eta / 20^{4} \\ \eta /(x+10)^{4} & 1 / 200^{2} & \eta /(x+10)^{4} & \eta / 20^{4} \\ \eta /\left(x^{2}+100\right)^{2} & \eta / 20^{4} & 1 /\left(x^{2}+100\right)^{2} & 0 \\ \eta /(x+10)^{4} & \eta / 200^{2} & \eta /(x+10)^{4} & 1 / 200^{2}\end{array}\right]$

$R S(127,63)$ coders are used by all of the transmitters. Working with the 2-state Markov chain fading model, we first calculate the threshold vector and the corresponding transition probability matrix as $\left[\begin{array}{lll}0 & 0.69304 & 9\end{array}\right]$ and

$$
\Pi=\left[\begin{array}{ll}
0.4085 & 0.5915 \\
0.3755 & 0.6245
\end{array}\right]
$$

respectively. The 3-state Markov chain model has a threshold vector of [ $\left[\begin{array}{lll}0 & 0.4054 & 1.0983\end{array}\right]$ and a transition probability matrix of

$$
\Pi=\left[\begin{array}{lll}
0.4688 & 0.5312 & 0 \\
0.5312 & 0.2588 & 0.3872 \\
0 & 0.3692 & 0.6308
\end{array}\right]
$$

Fig. 5 shows the curves of optimal total throughput versus $x$ the position of mobile node $A$. It includes two sets of curves associated with the two scenarios of known and unknown CSI. Each set of curves includes two curves associated with 2-state

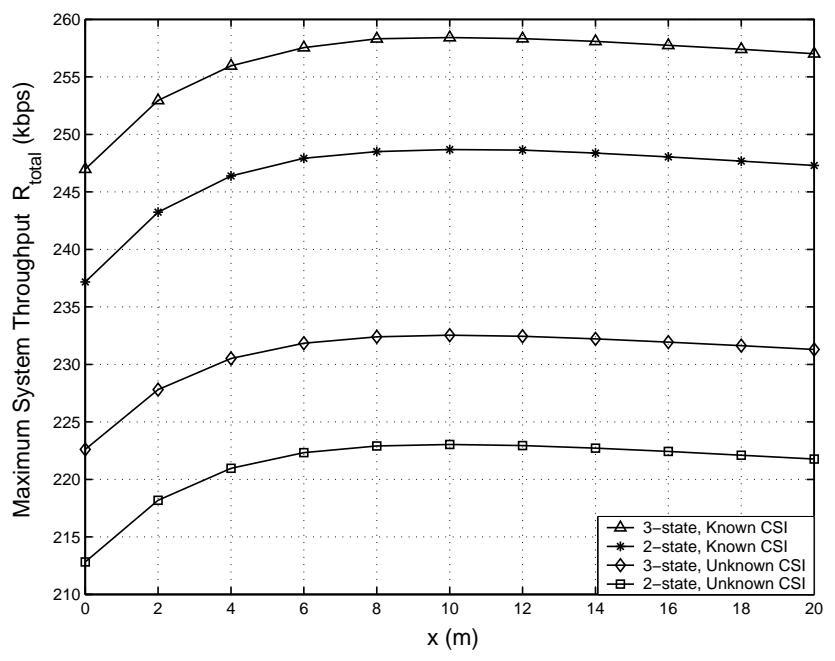

Fig. 5. Optimal curves of total throughput versus $x$ the position of mobile node $A$.

and 3-state Markov chain models. The most striking observation is the fact that all four curves are in the form of concave curves. While for the small values of $x$ the interference from other nodes reduces the overall throughput, for the large values of $x$ the throughput is decreased due to the loss of signal strength. The curves show that the throughput is at its maximum level when the value of $x$ represents the original position of node $A$ at $x=10$. It is also observed from Fig. 5 that the total throughput of the 3-state Markov chain model is better than of the 2-state Markov chain model in both sets. This is justified considering the fact that the 3-state Markov chain model is a more precise model of the Rayleigh channel than the 2-state model. Further, it is observed from Fig. 5 that having access to CSI provides better results in terms of throughput than not having access to CSI for similar 2- and 3-state models.

We have observed that an average of twenty and no more than thirty iterations are required for convergence. Finally, it is worth mentioning that while utilizing a 4-state Markov chain does not introduce a significant gain compared to a 3state Markov chain, it introduces significantly higher overhead of calculation. The results of the 4-state Markov chain model are close to those of the 3-state model and are not reported here.

\section{CONCLUSiON}

In this paper, we examined the problem of resource allocation in Rayleigh fading wireless ad-hoc networks with temporally correlated loss. Specifically, we sought to optimize the aggregate data rate of such networks subject to power and loss constraints. We analyzed fading statistics of signal-to-interference ratio (SIR), an important metric of transmission quality. Relying on our analysis, we modeled temporally correlated loss behavior of the Rayleigh fading wireless channel with finitestate Markov chains. We also noted that it would be straightforward to extend our approach to Ricean or Nakagami fading channels. We applied our Markov chain models toward solving our formulated throughput optimization problems. We also numerically validated our results by investigating our throughput maximization results under node mobility.

We are currently working on the integration of multiple antenna systems into our problem and analyzing their effects on our optimization problem. We are also investigating the implications of applying our results to the context of multimedia applications.

\section{REFERENCES}

[1] D. P. Bertsekas, "Nonlinear Programming, 2nd Edition," Athena Scientific Publishing, ISBN 1886529000, 1999.

[2] M. Chiang, D. O’Neil, D. Julian, S. Boyd, "Resource Allocation for QoS Provisioning in Wireless Ad Hoc Networks," In Proc. of IEEE GLOBECOM, 2001

[3] S. T. Chung, A. J. Goldsmith, "Degrees of Freedom in Adaptive Modulation: A Unified View," IEEE Trans. on Communications, September 2001.

[4] E. O. Elliott, "Estimates on Error Rates for Codes on Burst-Noise Channels," Bell Syst. Tech. J., vol. 42, September 1963.

[5] M. Hayajneh, C. T. Abdallah "Performance of Game Theoretic Power Control Algorithms for Wireless Data in Fading Channels," In Proc. of IEEE GLOBECOM 2003.

[6] W. C. Jakes, "Microwave Mobile Communications," John Wiley Publishing, ISBN 0780310691, 1994.

[7] D. F. Shanno, "Conditioning of Quasi-Newton Methods for Function Minimization," Mathematics of Computing, Vol. 24, 1970.

[8] C. C. Tan, N. C. Beaulieu, "On First-Order Markov Modeling for the Rayleigh Fading Channel," IEEE Trans. on Communications, December 2000.

[9] H. S. Wang, N. Moayeri, "Finite-State Markov Channel: A Useful Model for Radio Communications Channels," IEEE Trans. on Vehicular Technology, Februrary 1995.

[10] H. Yousefi'zadeh, H. Jafarkhani, M. Moshfeghi, "Power Optimization of Wireless Media Systems with Space-Time Block Codes," Submitted for Publication, 2003. Available at http://www.ece.uci.edu/ ${ }^{\sim}$ hyousefi/publ/powerIP.pdf 